\title{
EDUCACIÓN Y FORMACIÓN DE INTÉRPRETES DEL ARPA DE PEDALES EN COLOMBIA Y ESPAÑA EN EL SIGLO XXI
}

\section{Education and training of pedal harpists in Colombia and Spain in the twenty-first century}

\author{
Mónica Gallego López \\ Universidad de Burgos \\ Correo-e: mgallegol@yahoo.com.mx
}

Recibido: 20 de octubre de 2018

Envío a informantes: 3 de noviembre de 2018

Aceptación definitiva: 9 de abril de 2019

Resumen: Este trabajo brinda un acercamiento a los arpistas de pedales (maestros, intérpretes y alumnos) en Colombia y España, y ofrece una visión de la formación de los arpistas en el siglo xxi. Se realiza un acercamiento cualitativo a los arpistas en la actualidad con el diseño, desarrollo, análisis y evaluación de entrevistas, y participación en importantes eventos musicales, culturales y pedagógicos en torno al arpa de pedales, en los respectivos países. Se ofrecen significativas reflexiones en torno a la educación musical, $\mathrm{y}$, en especial, diferentes puntos de vista sobre la formación de los arpistas y el desarrollo profesional y educativo, convirtiéndose en un estudio pionero. La investigación muestra las aplicaciones que de la historiación crítica del arpa pueden derivarse para la formación de arpistas y el reconocimiento de su valor.

PALABRAS ClAvE: formación musical; educación; arpistas; desarrollo cultural.

AвsтRAct: This paper provides an approach to pedal harpist (teachers, interpreters and students) in Colombia and Spain, and offers a point of view of the harpist training in the twenty-first century. A cualitative approach is made to the harpist nowadays with the design, development, analysis and evaluation of interviews, and participation in important musical, cultural and pedagogical events around the pedal harp in the respective countries. Significant reflections on music education are offered, specially, different points of view on the harpist training and the profesional and educational development, 
becoming a pioneering study. The research shows the applications that critical history of the harp can be derived for education of harpist and the recognition of their value.

KEY WORDS: musical formation; education; pedal harpist; cultural development.

\section{Introducción}

$\mathrm{P}$ ARTIENDO DEL INTERÉS MUSICAL, PERSONAL y PROFESIONAL hacia el arpa, y como parte de las oportunidades de búsqueda de información sobre el tema de estudio -el arpa de pedales-, he decidido llevar a cabo un acercamiento a los arpistas, tanto en mi país de origen -Colombia- como en España, país de acogida para el trabajo de investigación. De esta manera, y por medio de una serie de entrevistas con sus respectivos cuestionamientos, he llevado a cabo este trabajo, con miras a vislumbrar cómo ha sido el desarrollo del arpa de pedales y la formación de sus ejecutantes, maestros y alumnos. Este panorama es tan solo un acercamiento a los profesionales en activo, jubilados, docentes y estudiantes a través de dos actividades musicales importantes que vienen sucediendo en estos países, en torno al instrumento. Al ser la primera vez que se realiza este acercamiento, en ambos países, es importante aclarar que no pretende ser, en ningún momento, un estudio de los sistemas educativos en Colombia y España, ya que requeriría de mayor tiempo de análisis e investigación; lo que pretendemos es realizar este acercamiento a los arpistas de pedales, aprovechando el acontecimiento de estas dos actividades musicales que tienen gran interés y relevancia en ambos países, para tener un panorama de la actividad actual de los arpistas de pedales.

En primer lugar, he realizado el acercamiento al objetivo de estudio: los arpistas de pedales, por medio de la participación en las principales actividades culturales que se vienen desarrollando actualmente en ambos países, como son: las clases maestras de arpa en Colombia y el Concurso "Arpa Plus» en España -los cuales se detallan más adelante-. En segundo lugar y teniendo en cuenta el estudio de recientes investigaciones en música, como el llevado a cabo por Bennett (20I0), en relación con la profesión de los músicos clásicos; considero de gran importancia llevar a cabo este acercamiento a los arpistas de pedales, para poder tener un panorama de cómo ha sido el desarrollo del instrumento, la formación de los arpistas y el desempeño actual de los mismos en el s. Xxi. Para llevar a cabo este acercamiento, se ha elaborado y aplicado como instrumento de investigación una serie de preguntas previamente elaboradas (Gallego López, 20I7) y validadas por expertos a manera de entrevista formal y llevadas a cabo con maestros, intérpretes y alumnos de arpa en sus entornos musicales, laborales y culturales; las cuales me han permitido corroborar aspectos inherentes a la educación de los arpistas como:

- Identificar las escuelas de arpa y conocer su desarrollo pedagógico y musical.

- Saber si los licenciados profesionales en interpretación musical -arpa-, que tienen un trabajo a tiempo completo, se desempeñan como arpistas en orquestas o en un conjunto instrumental de cámara.

- Conocer cuántos puestos de interpretación para arpa a tiempo completo existen y las posibilidades de conseguir dichos trabajos.

- Saber si la formación de interpretación a nivel orquestal ha existido por intérpretes nacionales o extranjeros y qué nivel de formación es brindado actualmente. 
EDUCACIÓN Y FORMACIÓN DE INTÉRPRETES DEL ARPA DE PEDALES

EN COLOMBIA Y ESPAÑA EN EL SIGLO XXI

MÓNICA GALLEGO LÓPEZ

- Conocer cuántos arpistas se ven obligados a salir de su país para continuar el perfeccionamiento instrumental y si el alumno actual continúa buscando formación en interpretación en el extranjero.

- Indagar sobre el promedio de arpista ocasional que participa en uno o más conciertos al año y sobre las orquestas de temporada o a tiempo parcial que dan trabajo a los arpistas.

- Saber si existen orquestas que ofrecen una o dos plazas de arpa dentro de la orquesta, cuántas vacantes de arpa existen, en cuanto tiempo se liberan estas plazas y cuáles son los parámetros legales para la participación en estas vacantes.

- Conocer si es necesaria una titulación en interpretación en el mercado laboral y si se requiere de un título oficial en interpretación para ocupar una vacante en una orquesta.

- Saber si están preparados los arpistas para participar en convocatorias de vacantes de arpa a nivel internacional, si se considera la carrera como intérprete a tiempo completo viable hoy día y hasta qué punto las oportunidades laborales existen de forma continua para este instrumento.

- Cuestionar si la ejecución de ciertas piezas de extracción popular podrían ayudar en la consolidación de determinados aspectos técnicos inherentes a la ejecución del arpa de pedal.

En tercer lugar, y una vez llevado a cabo el proceso de ejecución de las entrevistas, se ha realizado el tratamiento y categorización de las mismas por medio de la utilización de la herramienta «Open Code», la cual permite realizar el análisis y la evaluación cualitativa del instrumento aplicado, mismas que han sido detalladas con significativos análisis de frecuencia que me permiten comprender el objetivo de estudio, hasta llegar a los resultados. En relación a las entrevistas, cabe anotar que no realizamos una distinción de jerarquía en las respuestas en este artículo (profesionales, maestros, alumnos), solo mencionamos el número de entrevistado. Asimismo, es importante aclarar que son pocos los arpistas entrevistados, ya que ambos eventos limitan el tiempo de las entrevistas, debido a la gran cantidad de actividades realizadas en tan solo un fin de semana, situación que no nos permitió realizar un mayor número de entrevistas; reitero la oportunidad de realizar un acercamiento, no un estudio formal.

A manera de conclusión, se permite la voz participativa de los entrevistados con sus puntos de vista, en conjunto con las reflexiones propias, a los entrevistados en España; y se brinda una reflexión sobre la formación de los arpistas en Colombia, al ser el país donde la autora realizó los primeros estudios sobre el instrumento y poseer conocimientos más amplios respecto al acontecer musical en torno al tema de estudio.

\section{Un acercamiento a los arpistas (maestros, intérpretes y alumnos). Antecedentes}

Partiendo de la realidad de la formación profesional o superior que se imparte en los principales conservatorios de estos dos países, se debe mencionar que, en Colombia, la formación superior musical que se ofrece a los arpistas está sustentada por los conservatorios de música, instituciones estas vinculadas a una universidad. En España, por el contrario, existe enseñanza superior no universitaria. Los conservatorios 
superiores son instituciones de enseñanza superior no universitaria, en donde las circunstancias laborales y de autonomía de gestión no son las universitarias, pero sí lo es el nivel de sus titulaciones. Cabe mencionar que el Real Decreto I027/20II, de is de julio, establece el Marco Español de Cualificaciones para la Educación Superior; en el cual se tomó la decisión de adoptar un marco comprensivo de cualificaciones para el Espacio Europeo de Educación Superior; siendo este modificado por el Real Decreto 96/20I4, de I4 de febrero, en el que se establece el Marco Español de Cualificaciones para la Educación Superior (MECES), al igual que el 1393/2007, de 29 de octubre, que establece la ordenación de las enseñanzas universitarias oficiales.

Sobre este punto antes mencionado de la educación superior en España, resulta interesante mencionar que, en el momento de realización de esta investigación, sucedieron varias manifestaciones verbales y públicas de músicos, compositores y profesores de España, las cuales permiten una visión sobre el acontecer musical actual. Tal es el caso de "Músicos unidos por un nuevo diseño de las enseñanzas superiores», quienes piden al Gobierno actualización y modernización de los estudios superiores de música en España.

En el artículo publicado en ABC.es (23-07-2015) en Madrid y que brinda una reflexión sobre la cultura, Antonio Muñiz comparte sobre la reunión llevada a cabo en la Real Academia de Bellas Artes de San Fernando: «Los ponentes reclamaron una regulación propia para las enseñanzas superiores de música que permita la plena equiparación de España con los países del Espacio Europeo de Educación Superior donde ya existe una legislación especifica. Esto permitiría que los centros de estudios superiores dejaran de regirse por normas propias de la enseñanza media y se homologasen a los estudios universitarios, algo que haría posible un nuevo sistema de selección del profesorado, donde "la calidad y la especialización de los docentes sea la principal condición para su ejercicio efectivo”». Además, la plataforma considera indispensable la «preservación de la autonomía de los centros». Asimismo, denuncian que los conservatorios carecen de cualquier tipo de independencia «para confeccionar el claustro y promover la innovación y renovación de un profesorado». Demandan que la administración central se comprometa a «realizar los cambios legislativos necesarios para reordenar el modelo y que las administraciones educativas regionales procedan a la suspensión de convocatorias de plazas docentes para estos centros». Como ejemplo del «gran retraso que acumula España frente a otros países», leyeron la protesta enviada por los representantes del consejo escolar de un conservatorio -no se especifica cuál- en la que se narraba la poca preparación del profesorado, que en algunos casos no llega a tener la titulación requerida, así como la injerencia de las administraciones públicas y la imposibilidad de expulsar a los profesores que no cumplen con su trabajo. La conversión de los conservatorios superiores en «universidades de la música» significaría también un impulso a la investigación musical y permitiría que impartiesen el tercer ciclo de las enseñanzas superiores que da acceso al Doctorado, algo para lo que ahora no están habilitados. Cabe anotar que el compositor Agustín Charles, director del conservatorio de Aragón, denunció que «la educación superior musical está desplazándose hacia escuelas privadas». Todos los intervinientes subrayaron que «España es un país lleno de talento, como demuestra el vigor de una nueva generación de músicos y estudiantes que pueden compararse sin rubor a los países de Europa». Cabe mencionar que, en España, cuando un centro ostenta la denominación de conservatorio es porque imparte una titulación oficial y esta titulación requiere de un 
nivel de titulación oficial; a su vez que esta titulación requiere de un nivel de titulación de profesorado, según la Ley 8/20I3, LOMCE, artículos 58 y III (BOE de Io de diciembre de 20I3). Asimismo, es importante aclarar que en la actualidad los centros superiores de enseñanzas artísticas pueden impartir los másteres en enseñanzas artísticas; estos son oficiales a través de los cuales se puede acceder al doctorado.

$\mathrm{Y}$, haciendo referencia a la situación de la música, como asignatura pendiente en las comunidades autónomas, la compositora Eva Gancedo comenta: «La ignorancia de algunos políticos que hacen leyes que en vez de dar horario (a la música), se lo quitan porque piensan que sólo sirve para crear músicos» (Europa Press, julio I220I6). Importante anotar también otras reflexiones que se generaron en los medios de comunicación electrónicos sobre el desempeño y liderazgo de los músicos en España, a saber:

Los músicos por lo general trabajamos mucho en grupo y por otra parte recibimos una educación muy individualista y competitiva. Esta disfunción entre el tipo de formación que recibimos y el entorno en el que realizamos nuestra labor hace que existan gran cantidad de conflictos que podrían ser resueltos con una formación adecuada en la que se trabajaran específicamente las conductas adecuadas para trabajar en grupo. Estamos viendo como se crea una nueva concienciación y sensibilización sobre la necesidad de renovar aspectos y criterios a valorar cuando se realizan procesos de selección para incorporar nuevos miembros, bien sea una orquesta, una banda o un centro educativo. Cada vez más nos damos cuenta que más allá de los aspectos técnicos que un nuevo miembro puede aportar al grupo, están otros como la actitud, el carácter y en definitiva la conducta.

Es importante saber que «el individualismo inherente a la modalidad de clase de instrumento se complementa, en el modelo conservatorio, con las prácticas de conjunto, que posibilitan la socialización musical» (Asprilla, 2015).

Las anteriores reflexiones son importantes para nuestro trabajo de investigación, ya que, de una u otra manera, coinciden con situaciones vividas también por los arpistas, docentes e intérpretes. Aun pese a estos momentos, el artista continúa su trabajo, reinventándose cada día y logrando poco a poco un trabajo en comunidad. Tal es el caso de los arpistas de pedales, a quienes nos referimos a continuación.

\section{I.I. Una visión de las clases maestras de arpa en Colombia y del Concurso "Arpa Plus» en España}

Dentro de las actividades en conjunto que se vienen realizando en estos países y que promueven la participación de los músicos en beneficio de sus carreras, en especial, la de los arpistas, queremos hacer alusión a las llamadas "clases maestras» o clases magistrales, y a los encuentros y concursos que permiten el contacto entre ellos mismos y el fortalecimiento a nivel interpretativo y pedagógico. Hemos centrado nuestra atención en algunos de los eventos que a este nivel vienen sucediendo en Colombia y España, y aprovechamos estos espacios para llevar a cabo nuestro trabajo de investigación, procediendo a la ejecución y desarrollo del mismo. De esta manera, realizamos las entrevistas, tanto a intérpretes, como maestros y alumnos, dentro de los espacios artístico-musicales que propician encuentros entre los arpistas. 
En Colombia, tuvieron lugar entre los días I6-20 de diciembre de 2015, en el Conservatorio de Música de la Universidad Nacional de Colombia, en la llamada «Semana del Arpa»-XIII clase maestra-, como parte del convenio con la Fundación Salvi (Colombia). Esta Fundación lleva varios años promoviendo el instrumento en el país y facilitando los encuentros musicales y pedagógicos entre los arpistas. La actividad favorece el aprendizaje musical del instrumento, a través de las clases maestras dirigidas por un arpista invitado -de renombre internacional-, ante el cual, los estudiantes -de todos los niveles y procedentes de varias ciudades del país- interpretan parte del repertorio trabajado con sus maestros. Muy seguramente se espera que estos alumnos puedan hacer un análisis del mismo, como también de su técnica, en beneficio de su desempeño como intérpretes. Al finalizar la semana, el arpista invitado ofrece un recital -gratuito y patrocinado por la Fundación-, el cual está abierto al público en general y participa en la entrega de las certificaciones de participación en el evento. En palabras de la coordinadora y maestra de arpa se realiza la actividad «para hacer de esto un proyecto que les sirva a todos los estudiantes, a todos los arpistas, para darles herramientas, sobre todo, es un proyecto pedagógico, para brindarles a ellos herramientas, para que tengan mejorías en su trabajo, no solamente los estudiantes sino los profesionales, porque sabemos que nosotros -los arpistas- no tenemos acceso a la información con tanta facilidad, como sucede en otros países».

En España, tuvieron lugar dentro del evento que lleva por nombre «Arpa Plus», «Concurso para estudiantes de arpa», el cual se convirtió en un proyecto a nivel nacional y sucede cada año de manera itinerante. Este concurso, que tiene su propia web en internet y figura como «Arpa Plus» (2017), menciona entre sus objetivos la «imparcialidad de los tribunales y la igualdad de condiciones entre los participantes», y como este, se realiza por niveles, los cuales están adaptados al «nuevo sistema educativo» (cuatro cursos de Grado Elemental y seis de Grado Medio, conforme a la LOGSE). Cabe anotar que la LOGSE es una ley que ya no está en vigor en España. Asimismo, destacamos que, según los objetivos de Arpa Plus, «no se permite la participación a los estudiantes de nivel superior, debido a una serie de normas que impiden la competencia en niveles inferiores a los cursados y matriculados». La actividad, que sucede durante un fin de semana, cuenta con la participación no solo de los alumnos, sino también de maestros, intérpretes y padres de familia, procedentes de varios lugares de España, quienes se congregan para escuchar los sonidos del arpa. Al finalizar, se realiza la entrega de las certificaciones a los participantes del evento y los premios a los concursantes ganadores de cada nivel; destacándose como premio principal la entrega de un arpa Salvi -trovadora sin pedales-, entidad que también patrocina el concierto de clausura, con la invitación de un joven arpista -bien sea nacional o internacional- que tenga una carrera de excelencia interpretativa y haya sido ganador de premios. Este arpista suele ofrecer un taller abierto a la comunidad participante del evento, como parte del trabajo musical. El concurso también ofrece una exposición de instrumentos y accesorios, que cuenta con la participación de alguna tienda musical a nivel nacional y de Salvi, procedente de Italia. Desde el punto de vista de los arpistas, maestros, docentes e intérpretes, «Arpa Plus» es importante en la medida que permite la integración de los arpistas, fortalece los niveles de participación en el entorno musical y propicia un ambiente de imparcialidad. Asistimos entonces al Concurso «Arpa Plus» del año 20I5, celebrado en el Conservatorio Profesional de Música de Valencia, con el objetivo de tener un primer acercamiento musical y contacto directo con los 
arpistas, considerando el año siguiente, 20I6, para la realización de las entrevistas en el XviII concurso, que tuvo lugar en el Conservatorio Profesional de Música de Murcia.

Ambos acontecimientos, sucedidos en los países mencionados, tienen en común el desarrollo de la actividad musical interpretativa de los arpistas y promueven el encuentro entre los mismos. Asimismo, cuenta con la participación de arpistas invitados de excelencia musical -como parte del patrocinio de la Fundación Salvi-, permitiendo un intercambio pedagógico y cultural. Es interesante ver como los eventos favorecen el fortalecimiento técnico e interpretativo de los alumnos, en la medida que estos se preparan para participar en ellos y, de acuerdo a sus experiencias, surgen nuevas motivaciones -en su mayoría-, en beneficio del aprendizaje continuo. Haciendo relación a los principales criterios comparativos destacamos que la «Semana del Arpa», en Colombia, sucede siempre en el mismo lugar (Bogotá), no es un concurso sino que ofrece clases magistrales para todos los niveles; mientras que «Arpa Plus» es un concurso itinerante, organizado para estudiantes de los grados elemental y profesional de la especialidad de Arpa, pero no para los de Grado superior.

\section{Aplicación, análisis, evaluación y resultados de las entrevistas a los arpistas}

En las entrevistas realizadas tanto en Colombia como en España, se seleccionó un número igual de entrevistados en cada país (6) entre maestros (jubilados y activos), alumnos (recién graduados), profesionales e intérpretes, para un total de I2 entrevistados. Las preguntas fueron organizadas en cinco secciones y se desarrollaron en un tiempo promedio por persona de cuarenta y cinco minutos, para un total de 36 preguntas. Realizamos un análisis cualitativo, partiendo de la transcripción de las grabaciones y organización de la información, datos que arrojaron respuestas de gran interés para nuestro trabajo de investigación y los cuales nos permiten concluir lo siguiente:

I. En relación a las escuelas de arpa: Autonomía, programas de estudio, actualización y modernización de los programas superiores, innovación y renovación de profesorado, plazas docentes, calidad y especialización de los docentes, preparación y titulación. Sabemos que:

I. Los conservatorios de música en Colombia suelen estar ligados a una universidad, mientras que en España no dependen de estas instituciones: «No, es totalmente independiente, a nivel docente y también no tenemos nada que ver en cuanto a nivel administrativo, son dos cosas distintas» (entrevistado 9); "No, de hecho, en España, los estudios musicales no están equiparados con los universitarios, salimos después de haber estudiado una carrera de catorce años con un título que no es equiparable al que tiene la gente que sale de la universidad» (IO). Al parecer, muchos de los arpistas en España no tienen conocimiento de los reales decretos -antes mencionados- que corroboran que el Título Superior de una enseñanza artística en España es equivalente a todos los efectos al Título de Graduado universitario.

2. La posibilidad de investigación musical para los arpistas es poca comparada con otras áreas musicales. En las instituciones vinculadas a una universidad, la formación en investigación está empezando a tomar fuerza. «No, damos solamente formación del instrumento» (I2). 
3. No existen datos actualizados de los arpistas: instrumentistas de orquestas, grupos de cámara, docentes, pero se han ido estableciendo listas de contactos o bases de datos, a través del desarrollo de actividades continuadas, como las «clases maestras», encuentros y/o concursos de arpa, permitiendo de esta manera el contacto directo entre los arpistas; de igual manera se da el contacto a través de los medios electrónicos. «Por ejemplo, la Asociación Española de Arpistas: los que estamos asociados tenemos nuestra base de datos, con los contenidos actualizados, pero no hay una base de datos de todos los arpistas de España completo, para acceder a la información» (Io).

4. Los centros de trabajo (orquestas, escuelas) -en su mayoría- exigen las titulaciones profesionales. Algunos centros de carácter privado podrían no exigirlas: «Orquestas que son privadas, o semiprivadas, creo que no... depende si lo quieren ellos imponer internamente, pero no es una regla... que les imponga el Gobierno» (3).

5. Los arpistas cuentan con una formación pensando en su práctica laboral y profesional sostenible, en la medida que se les ofrecen nuevas oportunidades como clases con arpistas de reconocida trayectoria artística, participación en concursos y encuentros de arpa. Desde el punto de vista de los maestros «se ha visto un buen desempeño, el resultado de la gente que ha salido, la gente que está trabajando, es muy satisfactorio» (8). Por otro lado, algunos de los alumnos comentan: «No mucho, en el conservatorio, no nos enseñan a enfrentarnos a la vida laboral, nos enseñan muchas veces simplemente a tocar» (Io). En la formación oficial existe la educación para ser arpista orquestal, pero no existe un programa de arpista pedagogo. Algunos de los arpistas que reciben una formación en vinculación con universidades «están tratando de desarrollar ejercicios de gestión cultural, de investigación, de musicología».

6. En la actualidad, la mayoría de los arpistas coincide en afirmar que NO reciben nuevas posibilidades de educación y formación encaminadas a la proyección laboral del siglo XXI, exceptuando las clases magistrales que les permiten el contacto con arpistas de otros países. Desde el punto de vista de los alumnos, ellos mismos deben buscar estas nuevas oportunidades: «Veo el mundo del arpa como bastante anclado en lo clásico, a mí que me gusta más la música... más popular, a lo mejor haría falta una pequeña parte del curso, dedicado a otras músicas, no solo a lo clásico» (Io); «Creo que estamos un poco anclados todavía en la misma escuela» (II).

7. La psicología de la interpretación u otras nuevas tendencias educativas NO suelen ofrecerse a los arpistas. «Deberían tenerse presentes ya que las técnicas están en función de la persona, y no la persona en función de la técnica, hay muchos casos, en los cuales hay gente que abandona su carrera, sencillamente porque no es comprendida» (2). Al parecer, en ambos países desde hace pocos años se están ofreciendo algunas materias optativas, como informática musical. Aun así, algunos maestros en España comentan: «Estas ramas quizá estarían más vinculadas a los estudios de musicología en la universidad que están completamente separados de nosotros, o sea, nosotros nos ceñimos a las prácticas instrumentales» (9).

II. En cuanto a la formación profesional: Empleo del tiempo, destrezas y técnicas que se les exigen para desarrollar y mantener una carrera profesional, entorno en el que se desenvuelven laboralmente, importancia de la educación y formación que se imparte en la actualidad. Los arpistas:

I. Poseen las características necesarias para llevar adelante una carrera profesional. Desde el punto de vista de los alumnos, «deberían darse herramientas más sólidas y pensando en el futuro». 
2. Para mantenerse en la profesión deben tener herramientas musicales sólidas, estudiar y actualizarse, ser curiosos, tener una disciplina constante y participar en eventos. Por otra parte, el maestro debería tener «una adaptación, de la pedagogía y de la psicología, al servicio del individuo» (2). Y tanto maestros como alumnos, «abordar nuevas tendencias musicales como por ejemplo la música contemporánea» (9); «mantener una técnica sólida y cuidarse a nivel físico y emocional».

3. Tienen que desarrollar constantemente cualidades y habilidades de interpretación y no interpretación que les permitan mantenerse en la profesión, por citar algunas: orden, disciplina, responsabilidad, autoexigencia, compañerismo, interacción grupal, dinamización con el público.

4. Consideran que los fundamentos filosóficos que sustentan los programas formativos de nivel universitario y los cursos de música clásica orientados a la interpretación reflejan los requisitos necesarios para mantenerse en la profesión en la medida que se actualizan y permiten una formación no solo musical, sino de manera integral, en especial a los alumnos, para «que ellos sean parte de una sociedad constructiva, puedan tener la capacidad de análisis, y que entiendan sobre todo que uno funciona en grupo, aunque estemos encerrados la mayor parte del día estudiando; es para un beneficio social» (3). Tener presente que «las enseñanzas teóricas se integren en el instrumento, entonces formamos un músico» (9). Se debería cuestionar que «hay poco pensamiento filosófico ahora de lo contemporáneo» (го).

5. En su mayoría, los arpistas profesionales y profesores de arpa opinan que la formación de los arpistas debería darse de manera más homogénea, con el objetivo que les permita establecer y mantener un programa de estudios que recoja las habilidades y cualidades necesarias para la práctica profesional. Los alumnos, sobre todo, deberían «estar conectados a la realidad del país, saber qué es lo que necesita el país o lo que necesitan ellos, para desarrollarse, en uno u otros países». Asimismo, «si un profesor no desarrolla su trabajo, no toca, o no investiga, no escribe... no avanza» (3). De igual manera, sería interesante «permitir que dentro de esas habilidades técnicas propias de cada nivel, se puedan introducir diferentes géneros musicales, incluso, fomentar mucho la creatividad del estudiante, que es algo también fundamental para el arpista» (I). Es importante que «la práctica individual tenga una interrelación con otros departamentos de estudio, para permanecer activos» (8). «iEs tema muy interesante!, los planes de estudio se dividen en los cursos que tenga cada grado, y aquí es donde se ve la habilidad del profesor, escogiendo las obras por niveles... que se adapten a cada alumno, a sus gustos y a sus posibilidades» (9). De otro lado, los alumnos piensan que hay que tomar de todos los profesores lo mejor: «Es lo que más me ha ayudado a hacer mi propia técnica, mi propia forma de tocar» (Io).

6. Tienen acceso a una formación musical, mas no han recibido otras alternativas que les permitan desarrollar su potencial o ampliar sus roles dentro de la industria cultural. En este campo «todo es muy empírico..., los arpistas en general tienen una mentalidad más bien abierta porque como nos toca hacer todo solos y defender nuestra idea de que el arpa es un instrumento igual que los demás, entonces, todos estamos tratando de abrir espacios» (3). «Hoy día, se tienen posibilidades tecnológicas que antes no se podían ni soñar, y esto tiene que aprovecharse e incluso enseñarles a los alumnos cómo podemos aprovecharlo, para acceder a más conocimiento y que haya más diversificación de todo» (9); "Hay que hacerle ver a la gente que el arpa puede hacer muchas otras cosas... Eso también implica que el arpista tenga que adaptarse 
a nueva música... hay que arriesgarse un poco, si queremos que la gente vea el arpa como un instrumento actual y accesible» (Io).

III. Sobre la proyección laboral: Oportunidades en educación y formación que tienen los arpistas en el siglo xxI. Conocemos que:

I. Los profesionales del arpa: intérpretes o educadores, NO tienen -o es escaso- el conocimiento sobre las industrias culturales como medios que les permita explorar una carrera más integral. "Yo creo que el que tiene ese conocimiento es porque lo ha buscado por sí mismo, y se ha interesado por ello, no hay nadie que te enseñe eso» (Io).

2. La mayoría de los arpistas que estudian interpretación del arpa tienen posibilidades de lograr un nivel de calidad, pero son una minoría los que pueden conseguir los pocos trabajos disponibles en interpretación. Los maestros deberían brindar a los alumnos «más herramientas para que ellos no se sientan frustrados, sabiendo que no van a ganar un concurso de orquesta, simplemente porque no hay vacantes, o tienen que esperar diez años o más a que alguien se jubile, y ese no es el ideal... entonces que ellos sepan que uno no tiene que ser, o uno puede ser arpista y seguir... y toda la vida ser arpista, sin tocar en una orquesta» (3). La situación en ambos países parece ser igual: «Todos los que salen... que acaban la carrera, están capacitados, ahora, el problema es que no hay tantos puestos de trabajo... si solamente se pusieran plazas de arpa en todos los conservatorios profesionales que hay en España, es que todos ya estuvieran trabajando» (8); «Pueden conseguirlo todos, todos pueden llegar a ese nivel, el problema es que no hay plazas» (Io).

3. Todos coinciden en afirmar que el plan de estudios debería ofrecer más amplitud de oportunidades, basado en los intereses y potenciales del estudiante y de manera más realista conforme al medio de desempeño actual. Se debe brindar «un estudio adaptado a la evolución del ser humano» (7). De igual manera, la falta de apoyo para el desempeño musical -en ambos países- es cada vez mayor: "La profesión de músico, en España, no sé en otros sitios, no está valorada» (8). La labor del docente es fundamental: «El profesor está obligado a estar primero al día de todas las obras que se publican, estar al corriente de lo que se está editando y cómo lo aplica en su plan de estudios» (9); «Nos educan para "tocar” simplemente, y nos deberían educar para enfrentarnos a la realidad» (IO).

4. NO existe una cifra oficial de puestos de trabajo a tiempo completo disponibles para los licenciados en interpretación musical-arpa. En Colombia, por ejemplo, «no siempre las vacantes son de tiempo completo... no van a tener un profesor de arpa a tiempo completo, porque ellos consideran que no se necesitan tantos arpistas, entonces, sí hay profesores de tiempo completo, y no uno, sino como seis, de violín, de piano... pero de arpa no» (3). En ambos países, la falta de oportunidades para el desempeño laboral de los arpistas es cada vez mayor. En España, existe la bolsa de trabajo, pero «los interinos están sin trabajar» (7). Antiguamente, «en las orquestas, solía haber dos arpas por orquesta, pero esa tendencia, digamos que en toda Europa, está bajando, cuando una de las dos arpas se jubila, solamente dejan una, y la otra ya va, incluso hay alguna orquesta, como en el teatro del Liceu de Barcelona, ya no hay arpa allá» (9).

IV. Respecto a la formación y trabajo interpretativo: Orquestas, conjuntos instrumentales de cámara, formación de nuevas orquestas, creación de vacantes de arpista. Se tiene conocimiento de que: 
I. En la actualidad, NO hay puestos de interpretación para arpa a tiempo completo disponibles en las orquestas, los pocos que existen ya están ocupados y/o son pocas las posibilidades de conseguir estos puestos. «Cada vez somos más, cada vez hay menos trabajo, entonces lo que hace cada uno es buscarse su propia forma de vivir» (Io).

2. Existen las orquestas de temporada o a tiempo parcial que dan trabajo a los arpistas. La participación de los llamados «arpistas ocasionales» en uno o más conciertos al año es de un promedio del 50\%. «Pasa en todas las profesiones hoy día, un día les toca un bolo aquí, otro allá, viven un poco así de oportunidades» (9); «Existen orquestas que contratan arpistas solo para determinados programas» (Io).

3. NO existen orquestas que ofrezcan dos plazas de arpa hoy día. En Colombia no existen orquestas con dos plazas oficiales para arpa, en España, en algún momento existió: «La orquesta del real teatro del Liceu era una de ellas, tenía dos arpas, yo creo que hasta, no sé... no llega ni a diez años que estaban dos arpistas, una se jubiló, quedó la otra, y luego la despidieron, sin motivo aparente, no solamente al arpa, sino a muchas otras personas de la orquesta, y no se ha rehecho esta plaza» (9).

4. La carrera como intérprete a tiempo completo NO está siendo muy viable hoy día. Podría llegar a serlo, «mientras uno sea emprendedor y pueda abrirse sus propios espacios laborales» (5); «Quiero creer que sí, porque es nuestro futuro pero... tanto más gente estudia, más gente sale para poder trabajar, pero menos puestos de trabajo hay» (8); «Sí, tiene que serlo, la intención es esta... siempre es para tener... un empleo a tiempo completo» (9); «Es viable hoy día, si no pretendes vivir de la música cien por cien» (Io).

5. Las vacantes de arpa existentes -tanto en las orquestas como en los conservatorios- son escasas, tanto en España como en Colombia. No existen actualmente. De igual manera, se tiene conocimiento de que dentro de los parámetros legales para la participación en estas vacantes están -entre otros- el ser profesional y tener experiencia en el campo.

En ambos países, estas plazas tardan mucho tiempo en ser liberadas: por ejemplo, en España «la gente cuando entra allí, entran más o menos, medianamente joven, la edad de jubilación aquí es sesenta y cinco o más, imagínate entrar a los treinta, se libera hasta dentro de treinta». Asimismo, «no hay tantísimas plazas de profesor de arpa... tienen que crear un montón, no solamente de arpa profesional sino de superior, se deberían crear, pero sobre todo de profesional, porque es la base, superiores hay bastantes, pero profesionales faltan muchísimo» (8).

6. Sí es necesaria una titulación en interpretación en el mercado laboral, especialmente en el medio oficial. Al parecer algunas instituciones, especialmente las privadas, suelen tener sus propias reglas «una vez, solamente una vez, en la orquesta de la ciudad de Barcelona, se prescindió de este requisito» (9).

7. Los arpistas SÎ́ están preparados para participar en convocatorias de vacantes de arpa a nivel internacional, pero es muy difícil acceder a estas, ya que generalmente se realizan por «invitación». "Es el deseo nuestro como profesores que los alumnos que salen formados puedan ir a cualquier sitio, con esa formación... por eso es muy interesante, completar la formación con algún arpista, una persona que haya estado en activo en estas labores, para que los aconseje» (9); «Si el arpista en España está preparado, es porque lo ha hecho particularmente, yo creo que poca gente sale preparada del conservatorio para afrontar una prueba y ganarla» (Io).

8. La gran mayoría de los arpistas $(80 \%)$ se ven obligados a salir de sus países para continuar el perfeccionamiento instrumental, pero también «hoy día no es que sea 
una obligación sino que es un gusto» (9). En Colombia lo hace la mayoría, mientras que en España «no hay muchos arpistas que se atrevan a irse fuera... hay poco porcentaje de arpistas fuera» (го).

V. Haciendo referencia de la formación a futuro y la profesión de las arpistas -docentes e intérpretes-, educación, formación y futuro laboral. Sabemos que:

I. Los intérpretes-docentes de arpa, en su mayoría, coinciden en responder que NO poseen las técnicas pedagógicas básicas o alguna cualificación docente. «En Colombia estamos viendo como que se desmerita un poco... esa parte del docente, entonces se preocupan mucho por que toquen bien, porque tengan unas competencias musicales, pero la parte pedagógica pasa a un segundo plano» (I). En algunos casos, «los arpistas han realizado prácticas de enseñanza». En España «No hay preparación docente en el conservatorio» (Io). Pero el intérprete, en términos generales, «se puede enfocar o bien a la parte instrumental o a la pedagógica». «El título superior, como instrumentista, te permite acceder a una plaza para enseñar y se supone que has tenido alguna asignatura relacionada con la enseñanza, pero algo muy específico, no, no hay una formación» (II).

2. Todos los arpistas afirman que el trabajo de otros géneros de música, diferente a la música clásica, puede brindar más oportunidades a nivel laboral, ya que «hay un mundo enorme por desarrollar» (3). Debería darse: «Sí, por supuesto, porque nuestra cultura, incluso, no es tan clásica» (I); «Por la necesidad de diversificar para mantenerse activo laboralmente» (5); «Cuanto más abierta está una persona, mejor» (9).

3. SÍ es importante tener conocimiento sobre música contemporánea, músicas del mundo o música digital para el trabajo laboral. «Es un enriquecimiento todo esto, sobre todo la música contemporánea, existen compositores actualmente que escriben para el instrumento normalmente muy desorientados, siempre tienen mucho miedo de escribir para arpa, y luego, el arpista ha de estar preparado para objetar alternativas en lo que él ha escrito... siempre para mejorar» (9).

4. NO es común el uso de las tecnologías musicales por parte de los arpistas del siglo xxI. Se considera «necesario» y se empieza a «tener presente», ya «hay gente que investiga» -en algunos casos-. La tecnología musical ha empezado a formar parte de las asignaturas optativas que se ofrecen en los conservatorios de música. «Muy necesario es el conocimiento, sobre todo en la escritura, es como cultura básica, también, acceder a todo tipo de grabaciones con toda facilidad» (9).

5. Se está empezando a tener presente la realidad de la profesión, de manera que se pueda reflejar en los programas orientados a la interpretación que existe en la actualidad. «Todo esto es trabajo del profesor, adaptarse» (9).

6. Se «deben» y pueden aprovechar las oportunidades de las industrias culturales para la búsqueda de empleo, formación, financiación y redes de contacto profesionales. Resulta ser «una falencia muy grande, que en algún momento, uno se siente a desarrollar» (3); «Muchas veces no corresponde esto, digamos, con realidades artísticas» (9); «Se pueden aprovechar si tienes muchísima suerte, y encuentras un contacto que realmente quiera apostar por ti, por tu trabajo, porque las instituciones públicas, ahora mismo, prácticamente no dan ni una ayuda económica, para tus proyectos, para tus conciertos» (Io).

7. NO existen orquestas suficientes en relación con el número de conservatorios y escuelas de música. «No, las orquestas están muy por debajo de la realidad» (9); «No 
hay muchas orquestas, algunas de ellas son amateur y otras son privadas, y, normalmente, no contratan arpistas» (Io).

8. El uso del teclado u otro instrumento electrónico para sustituir al arpa sigue sucediendo de manera ocasional: «Lastimosamente, se sigue dando eso» (I).

9. La interpretación $\mathrm{NO}$ es la única profesión que merece la pena para los licenciados de los conservatorios; ya que «hay un campo de acción enorme» $\mathrm{y}$ «muchos otros campos por explorar»; "La creatividad, la novedad y las iniciativas personales son demasiado importantes para la evolución de un arte»; «Definitivamente, estamos en un mundo que está cambiando mucho, que están surgiendo muchas formas distintas de concebir la profesión de músico, la interpretación como se entiende convencionalmente no es la única»; "Hay que interrelacionar lo que es la música del arpa con otras áreas, investigación, también»; «Es muy importante la interpretación porque es el objetivo de los alumnos, todos hemos ido a la interpretación, después, se diversifica según las salidas laborales que encuentras» (9); "Hay gente que busca la interpretación como medio de vida, otra gente que prefiere la enseñanza, y otra que prefiere la investigación» (Io).

Io. Actualmente, los gobiernos Sí suponen que los licenciados solo están preparados para seguir carreras profesionales en la interpretación "por falta de conocimiento» o «ni se ocupan de ello», «los gobiernos en música no piensan nada, es una asignatura que tienen pendiente, que no sé si se resolverá nunca» (9); «Supongo que los gobiernos piensan que no estamos capacitados para nada, porque no nos dan ninguna ayuda... a lo mejor alguien muy, muy virtuoso, que haya ganado algún premio de gobierno o premio público, es alguien a quienes ellos puedan promocionar, pero al resto de músicos, en general, no los valoran en absoluto» (Io).

II. Los arpistas coinciden en afirmar que la mayoría de los conservatorios están sometidos a la presión de formar únicamente para carreras profesionales en la interpretación. «Por ahora, las únicas posibilidades claras, a las que el conservatorio se puede comprometer a educar un músico, es como intérprete» (5). "Sí, creo que... está clarísimo, que el objetivo de los conservatorios es ese, formar profesionales, y los propios alumnos son los que más condicionan esta salida» (9); "Puede ser que el programa les exija a los profesores mucha más dedicación a la interpretación» (Io).

I2. La especialidad de arpa en la formación musical dentro de los planes de estudio a nivel educación primaria y/o secundaria NO existe de manera oficial. En ambos países, se ofrece en algunas entidades privadas -en su mayoría- como actividad extracurricular: «En España hay arpa en actividades extraescolares». A nivel oficial se tiene conocimiento de un centro educativo en Madrid «donde están combinando la educación primaria y secundaria con el conservatorio» (Io). «Las enseñanzas generales de los colegios, cada vez más, van reduciendo las clases de música, o sea, es una formación que se debe potenciar y ya no solamente desde los conservatorios para las personas que deciden dedicarse profesionalmente, sino en los colegios. La formación musical debería formar parte de los currículums de las enseñanzas, luego el que quisiera profundizar, o que vea que eso le puede interesar para dedicarse profesionalmente, ir a un conservatorio. Es una asignatura pendiente que tenemos como sociedad y cultura».

En relación a este último punto es importante anotar que no todos los entrevistados poseen conocimientos actualizados concernientes a la pregunta. Debemos aclarar que en España la enseñanza oficial de un instrumento no está incluida en los 
centros de primaria y secundaria. A lo que se refiere lo anterior es a los Centros Integrados de Música (CIM) que combinan las enseñanzas de régimen general y régimen especial (música) en un único centro. En concreto en Madrid, existen dos centros de estas características; son centros públicos y oficiales (сім Moreno Torroba de Madrid y сім Padre Antonio Soler de San Lorenzo del Escorial).

Cabe anotar otras reflexiones hechas por los entrevistados -a manera de conclusiones- respecto al desempeño de los arpistas en España, a saber:

I. En cuanto a la formación: «La música en España, desgraciadamente, no está muy situada en ningún sitio, en elemental, se supone que tendría que estar en la escuela; en la primaria, no lo está; en enseñanzas profesionales, nos equiparan a secundaria, que tampoco tiene por qué ser, porque una enseñanza secundaria es obligatoria, la enseñanza de música es una enseñanza de régimen especial y tú la escoges porque quieres realmente; en cuanto a la enseñanza superior, es solo eso, superior, no es universitario, no estamos dentro de la universidad»; «Un estudiante que termina el superior, debe buscar un título universitario en el exterior, porque no lo encuentran acá» (7). En relación a este punto, muy seguramente el entrevistado quiso referirse a estudios de postgrado en el instrumento, maestrías o doctorados, títulos estos inexistentes hasta la fecha de las entrevistas en España. «La educación en música se rige por las leyes de institutos de secundaria y las enseñanzas especiales; nos gustaría, por ejemplo, que los superiores sí que estuvieran ligados a una universidad, ¡es fundamental!». Nos preguntamos entonces, la titulación que recibe un arpista en España, en el último grado -superior-, ¿puede ser igual a una licenciatura universitaria?

2. En relación a los puestos de trabajo en España, se sabe que «depende de las comunidades, ya que son autónomas». Por ejemplo, en la Comunidad de Valencia, los arpistas que tienen un puesto oficial o «plaza» a tiempo completo en un conservatorio no pueden ejercer -tiempo completo- como arpistas de orquesta, lo pueden hacer de manera parcial. «No puedes tener jornada completa en un conservatorio, por lo menos en Valencia, y estar en una orquesta, no es compatible» (7). Asimismo, existen las bolsas de trabajo: «Una vez salen las convocatorias, te presentas a la oposición y hay selección, por ejemplo, en la orquesta de Valencia hicieron pruebas, entonces hay un orden y llaman a la primera de la bolsa, si no a la segunda». "No son más de is las plazas oficiales en España».

3. En cuanto al programa de estudios, se menciona que existen evaluaciones de los mismos por parte de las entidades encargadas, como también cierta autonomía: "Yo presento mi programa de estudios, es estudiado por el centro -conservatorio-, este lo lleva a inspección en la Consejería de Educación y si es apto lo aplico y desarrollo, pero hay libertad, el programa puede variar, en los últimos años varias de las arpistas estamos tratando de unificar criterios y niveles». El intento de homogeneidad «sí existió, en el pasado, en el año 66 se hizo una planificación, por aquel entonces solo había dos conservatorios en arpa, en Madrid y Barcelona, entonces, se unificó, pero esta duró cuatro días, porque ya fue el propio Madrid el que se independizó totalmente con el programa, y luego en Barcelona se estaba siguiendo un programa que nadie respetaba y de esta manera, cada uno se fue por su lado, pero resulta ser una cosa bastante común, en líneas generales, no nos podemos separar mucho porque tampoco es que tengamos el "gran programa” en arpa, no, pero también es bonito que cada centro tenga un poco su propio perfil» (9). Cabe anotar que las aprobaciones de las 
programaciones didácticas están sujetas a que se cumpla la normativa correspondiente, estatal y autonómica, establecida para los diferentes planes de estudio.

4. Respecto a la enseñanza del arpa en el siglo xxi se tiene conocimiento de la existencia «a nivel superior», del perfil Jazz (incluye música ligera) y el perfil Barroco, que existe en España, además del perfil Clásico que ya conocemos y el de Jazz aún no se ha implementado en ningún conservatorio (está solo contemplado en la ley).

5. Existen clases de arpa en centros privados en los que los estudios son oficiales (con reconocimiento de las autoridades autonómicas), tal es el caso del Centro Superior Katarina Gurska, en Madrid.

Haciendo referencia a los arpistas en Colombia, podemos concluir que:

I. La formación de los arpistas se da por niveles y se opta al título profesional ofrecido por los conservatorios que están ligados a una universidad.

2. Solo existe I puesto oficial o «plaza» de arpista a tiempo completo, las otras opciones de trabajo existentes -pocas, actualmente- se dan por contrato o se usan los llamados arpistas invitados u «ocasionales»; sucediendo igual a nivel docente.

3. Los programas de estudio son autónomos.

4. Existen clases de arpa en muy pocos centros privados y fundaciones que ofrecen estudios sin reconocimiento oficial.

\section{Reflexiones sobre la formación de los arpistas en Colombia}

El quehacer musical en Colombia, en especial el que se refiere a la interpretación musical del arpa, ha tenido su espacio -en su mayoría- en los conservatorios o escuelas de música que forman parte de una universidad. Asimismo, ha obtenido sus bases de modelos educativos tomados de otros países, en donde realizan estudios los arpistas que tienen la oportunidad de salir a perfeccionarse en el instrumento y los cuales son aplicados por estos a nivel nacional. Estos «formados» arpistas, una vez regresan al país, pueden ejercer como ejecutantes de arpa en una de las pocas orquestas que existen y/o generalmente optan también por ser «maestros» de algún conservatorio o escuela de música, siendo muy pocas las instituciones musicales que ofertan la cátedra de arpa. La formación que brindan estos «maestros ejecutantes» a sus alumnos está basada en los modelos extranjeros que aprendieron en su etapa como intérpretes, mas no sustentan -en su mayoría- una formación con bases pedagógicas, por lo tanto, debería darse una mediación educativa que permita un intercambio de aprendizaje en pro del desarrollo pedagógico musical, sin limitarse a la aplicación de la rigidez técnica, en especial, dentro del entorno interpretativo y cultural. Los pocos conservatorios que ofrecen la cátedra de arpa en el país presentan diversos modelos didácticos, por lo tanto se observan diferencias importantes en cuanto a propósitos de enseñanza, contenidos, estrategias, metodologías didácticas y de evaluación, ideas e intereses de los estudiantes y relación con los contextos sociales. Podríamos decir que prevalecen las prácticas ancladas a la tradición, centradas en la preparación de repertorio fundamental a través de la imitación y la repetición, y con poca atención a los intereses de los alumnos. Por lo tanto, resultaría interesante brindar una educación con mayor énfasis en las necesidades de los estudiantes y las limitaciones del medio actual, en donde cada vez más los espacios artísticos se reducen, al igual que el apoyo hacia los artistas, 
del mismo modo que resultan escasas las orquestas e instituciones educativas que posibiliten la apertura y difusión del instrumento, bien sea por falta de recursos o por inadecuadas políticas directivas e institucionales. Debería darse un enfoque educativo hacia el siglo XXI, en donde los músicos-arpistas puedan ser también sus propios gestores y productores musicales.

La educación musical debería ser un proceso integral, por ende, es importante que la práctica de los arpistas en Colombia se encamine a una formación lo más humanamente posible, en donde el proceso educativo se construya con base en las necesidades del alumno, sin dejar de lado su idiosincrasia y sus limitantes concebidas en el entorno natural y cultural. Desde nuestro punto de vista como ejecutantes, pedagogos e investigadores musicales, y tomando como punto de partida varias de las investigaciones realizadas en el campo musical, es importante cuestionarnos sobre los pensamientos de profesionales involucrados activamente en el proceso del desarrollo de la educación musical, los cuales de una u otra manera también deberían estar presentes en nuestro entorno musical nacional, como son:

I. ¿Es la música, por encima de todo, un arte social en el que la interpretación con otros y la escucha de otros es la motivación, la experiencia y el proceso de aprendizaje?

2. ¿La educación musical por el encuentro contrasta con la instrucción musical y las estrategias de enseñanza de habilidad mixta?

3. ¿El profesor eficaz es sensible a las necesidades de los alumnos y a la estructura y naturaleza de la asignatura?

A lo largo de nuestros años de experiencia en la labor musical, bien sea dentro de la ejecución instrumental del arpa o de la pedagogía musical, han surgido varios cuestionamientos sobre la educación musical en Colombia y, en especial, la referente a los arpistas. Asimismo, ante la poca información respecto a nuestro tema de estudio, hemos querido llevar a cabo una búsqueda de información, encaminada a saber cómo ha sido el desarrollo de la práctica del arpa de pedales y, por ende, la formación de los arpistas. Un estudiante de arpa aspira a desarrollar una carrera como concertista o músico de orquesta o de cámara. Sin embargo, las probabilidades son pocas, sobre todo a nivel orquestal donde la mayoría de las orquestas en el mundo solo ofrece una plaza para arpa. De este modo, gran parte de los arpistas deben dedicarse a la enseñanza del instrumento en escuelas de música y conservatorios; siendo difícil lograr también una plaza en una institución de importancia musical donde el arpa no es considerada aún hoy día, sobre todo en Latinoamérica, como un instrumento importante. En Colombia, los arpistas, sujetos a las opciones de trabajo existentes, dedican su tiempo para ejercer otras funciones diferentes a la interpretación. Los pocos arpistas profesionales que existen en el país -quienes deberían tener un trabajo que les permita desempeñarse como intérpretes- compaginan su labor de interpretación con la enseñanza, siendo muy pocos los que poseen, además, una cualificación docente. Las oportunidades laborales para los arpistas dentro de las orquestas, nos atrevemos a decir, han sido desfavorables. En primer lugar, porque hasta hace muy poco -segunda mitad del siglo xx-surgieron las llamadas «plazas» o posiciones oficiales para arpa y, en segundo lugar, porque son pocas las orquestas existentes que brindan este tipo de "plaza». De otro lado, desde la formación de las primeras orquestas en el país -siglo xx- hasta la fecha, se han dado inicios y términos de este tipo de agrupaciones, de manera que no permite que exista una continuidad laboral para los músicos y mucho menos para los arpistas. Por ejemplo, son varios los casos de arpistas nacionales que han trabajado 
temporalmente en el país sin posibilidad de continuación laboral, ya que se ven en la obligación de salir del mismo, debido a la disolución de estas agrupaciones, que en su mayoría han terminado por «falta de presupuesto», o por cambios dados en el «gobierno de turno» o a nivel «institucional». Asimismo, la concepción del instrumento como parte importante dentro de la orquesta sigue siendo arbitraria, bien sea por capricho de algunos «directores de turno", quienes deciden sustituirla muchas veces por el teclado o porque sencillamente no han permitido que forme parte de la orquesta con una plaza oficial, con la excusa de «falta de recursos» para pagar al arpista o para comprar un instrumento. Aun así, han sido varios los arpistas que han puesto su propio instrumento a disposición de estas agrupaciones y algunas veces también de las instituciones musicales, con el deseo de darle mayor difusión y tener una posible oportunidad laboral, pero algunas veces se han llevado malos ratos, debido al mal manejo o movilización de sus instrumentos, los cuales, en algunas ocasiones, han sido maltratados o dañados, por la falta de conocimiento del instrumento y de la manera como debe transportarse. Podríamos decir entonces que la falta de conocimiento hacia el instrumento y muchas veces también la apatía por parte de algunos directores y gobiernos continúan dándose en el país.

Es bien sabido que, en la actualidad, la mayoría de orquestas en el mundo han implementado cambios dentro de su cronograma de actividades o programación musical y consideran el poder llevar la música a la comunidad, por medio de conciertos didácticos en escuelas, o realizar conciertos de temporada en iglesias, plazas y parques. Bennett, haciendo reflexión sobre la educación y el trabajo hacia la comunidad que ofrece el Berklee College of Music, comenta como "pone énfasis en el desarrollo de carreras en la profesión de la música en su totalidad y esto se refleja en un plan de estudios ecléctico y unos programas comunitarios envidiables como el programa City Music, que proporciona educación musical gratuita para los jóvenes de las zonas urbanas deprimidas de Boston». Modelos como los anteriores y otros más también han sido llevados a cabo en Colombia, permitiendo un mayor acercamiento de la cultura al pueblo, a un aprendizaje y a una mayor valoración del arte en nuestra sociedad. Pero aun existiendo estas oportunidades en el medio musical nacional, muchas veces la figura del arpa continúa siendo relegada al teclado con sonido de arpa por parte de algunas orquestas o instituciones. ¿Hasta qué punto las oportunidades laborales existen de forma continua para este instrumento?

La formación del arpista en Colombia debería partir de la realidad social y cultural, de manera que le permita al estudiante un mejor enfoque de su desarrollo profesional. De igual manera, el maestro debería brindar desde el principio un aprendizaje integral, en donde el alumno desarrolle su capacidad de mente abierta. "Los alumnos aprenderán más cuando se sitúen en experiencias complejas donde sean libres para procesar, analizar y examinar la experiencia de significado y comprensión y donde puedan relacionar lo que han aprendido con su propio objetivo» (Boardman, 20or). Una educación musical en interpretación del arpa que pueda integrar otras ramas profesionales como medio de apoyo, por ejemplo, las relacionadas con industrias culturales, resultaría más eficaz en la medida que proporciona más elementos educativos con miras hacia el futuro. Los programas de música en el país deberían implementar cambios que permitan una mayor proyección laboral, en especial los que tienen que ver con la formación de los arpistas. Es fundamental formar a las personas, en este caso a los músicos-arpistas, con una actitud empresarial desde el primer momento. En 
nuestro trabajo de evaluación de las entrevistas realizadas, hemos podido confirmar que no se brinda a los arpistas una formación con miras al desarrollo y desempeño laboral en el siglo xxi. Resulta escaso tanto por parte de los maestros, intérpretes y alumnos el conocimiento de las industrias culturales, los programas tecnológicos musicales o la psicología de la interpretación, por mencionar algunos. Asimismo, es limitante el repertorio seleccionado -en su mayoría-. El 90\% de las obras trabajadas dentro del repertorio corresponden a piezas clásicas, siendo muy pocos los maestros que han llegado a considerar dentro de su programa alguna obra del repertorio de su país o de otros, de manera que les permita desarrollar un poco más el trabajo interpretativo y enriquecerlo con nuevos elementos musicales y creativos, en beneficio del trabajo de creación musical de sus alumnos, que resultaría interesante para el fortalecimiento de la búsqueda de identidad musical propia. Es importante que no existan barreras de aprendizaje. Un alumno libre de fronteras realiza un crecimiento integral a través de las actividades transversales que pueden ayudar a su desarrollo cultural y artístico, en este caso, de los arpistas. Asimismo, el repertorio no debería limitarse al trabajo clásico, permitir la exploración de nuevas músicas fortalece el trabajo interpretativo y laboral. Dentro de la experiencia personal, podríamos compartir varios logros y satisfacciones musicales en donde el desarrollo de la creación musical y la inclusión de elementos regionales y de música del mundo han estado presentes en las nuevas prácticas artísticas.

Es importante entonces que las instituciones educativas preparen al estudiante para enfrentar una realidad cada vez más aglomerada de músicos intérpretes, en este caso, hacemos referencia a los arpistas, los cuales van a encontrar cada vez menos posibilidades a nivel laboral en las orquestas y como solistas en grandes salas de conciertos.

\section{Conclusiones}

El panorama de los intérpretes del arpa de pedales, tanto en España como en Colombia, es limitado con relación a los avances del s. Xxi. La mayoría de los arpistas entrevistados (90\%) afirman que las posibilidades de educación y formación no se encaminan conforme a la proyección laboral actual. La formación de los arpistas es condicionada por los gobiernos e instituciones oficiales, por lo tanto, el plan de estudios debería ofrecer más amplitud de oportunidades, basado en los intereses y potenciales del estudiante y de manera más realista conforme al medio de desempeño actual. Los intérpretes del instrumento no poseen los conocimientos necesarios sobre las industrias culturales como medios que les permitan explorar una carrera más integral y la gran mayoría de los arpistas $(80 \%)$ se ven obligados a salir de sus países para continuar el perfeccionamiento instrumental.

Las oportunidades laborales son escasas. En la actualidad, no existen puestos oficiales de trabajo a tiempo completo para los arpistas en ambos países, tanto a nivel orquestal como docente. La práctica instrumental es limitada y no existen orquestas suficientes en relación con el número de conservatorios y escuelas de música.

Por otra parte, los encuentros y concursos de arpa permiten el desarrollo de la actividad musical interpretativa de los arpistas y promueven el encuentro entre los mismos. 


\section{Bibliografía}

Arpaplus (20II). Concurso Arpa Plus. España. Disponible en: http://www.arpaplus.org.

Arthurs, A. (2004). Why Creative Industries. Australian Music Forum, ro(5). Australia: Autor. Asprilla, L. I. (20I5) . Educar en la música: Una aproximación crítica al talento y la educación musical. Revista Aula, 2I, 63-83.

Bennett, D. (20Io). La música clásica como profesión. Pasado, presente y estrategias para el futuro. Colección Crítica y Fundamentos, 29. Trad. Jiménez, B. Barcelona: Editorial Graó.

Boardman, E. (200I). The Relationship of Musical Thinking and Learning to Classroom Instruction. En E. Boardman (ed.), Dimensions of Musical Learning and Teaching: a Different Kind of Classroom. Reston, va: Autor.

Gallego López, M. (20I7) Disponible en http://riubu.ubu.es/handle/ı0259/4777.

Gancedo, Eva (20I6, I2 de julio). La Música, asignatura pendiente en las comunidades autónomas. Europa Press.

Muniz, A. (2015, 23 de julio). ABC.es. Regulación enseñanzas superiores de música en España. ABC.es.

SAlvi (20I5). Disponible en: http://www.fundacionsalvi.com. 
